\title{
REVIEW ON VEHICULAR SPEED, DENSITY ESTIMATION AND CLASSIFICATION USING ACOUSTIC SIGNAL
}

\author{
Prashant Borkar ${ }^{1}$, Latesh G. Malik ${ }^{2}$ \\ ${ }^{1,2}$ Department of Computer Science and Engineering, G.H. Raisoni College of Engineering, Nagpur, \\ India
}

Received 20 October 2012; accepted 28 March 2013

\begin{abstract}
Traffic monitoring and parameters estimation from urban to non urban (battlefield environment) traffic is fast-emerging field based on acoustic signals. We present here a comprehensive review of the state-of-the-art acoustic signal for vehicular speed estimation, density estimation and classification, critical analysis and an outlook to future research directions. This field is of increasing relevance for intelligent transport systems (ITSs). In recent years video monitoring and surveillance systems has been widely used in traffic management and hence traffic parameters can be achieved using such systems, but installation, operational and maintenance cost associated with these approaches are relatively high compared to the use of acoustic signal which is having very low installation and maintenance cost. The classification process includes sensing unit, class definition, feature extraction, classifier application and system evaluation. The acoustic classification system is part of a multi sensor real time environment for traffic surveillance and monitoring. Classification accuracy achieved by various studied algorithms shows very good performance for the 'Heavy Weight' class of vehicles as compared to the other category "Light Weight". Also a slight performance degrades as vehicle speed increases. Vehicular speed estimation corresponds to average speed and traffic density measurement, and can be substantially used for traffic signal timings optimization.
\end{abstract}

Keywords: acoustic signal, noise, speed, density, vehicle classification.

\section{Introduction}

Traffic congestion has significant impact on both economy and environment. Traffic congestion has been increasing worldwide due to motorization and urbanization. This results in increasing usage of vehicles worldwide and vehicles $\mathrm{CO}_{2}$ emission is becoming the main reason for global warming ( $\mathrm{Li}$ and Shimamoto, 2011). Reducing such traffic congestion can significantly improve traffic flow, reduces travel time and the environmental impact. Traffic architectures mainly depend on an infrastructure of sensors capable of automatically monitoring traffic conditions. Automatic determination of traffic congestion status is thus introduced to reduce the cost of human resource and the traffic congestion delay.

In recent years, there has been an increased scope for the automatic analysis of urban traffic activities and also for battlefield environment. This case is due, in part, to the additional numbers of cameras and other sensors, enhanced infrastructure, and consequent accessibility of data. In addition, the advancement of acoustic signal processing together with increased computing power has enabled new applications.

${ }^{1}$ Corresponding author: prashant.borkar@raisoni.net 
Traffic density on roads and highways has been increasing constantly in recent years. Traffic in developed countries is characterized by lane driven. Use of magnetic loop detectors, video cameras, and speed guns proved to be efficient approach for traffic monitoring and parameter extraction but the installation, operational and maintenance cost of these sensors significantly adds to the high operational expense of these devices during their life cycles. Therefore researchers have been developing several numbers of sensors, which have a number of significant advantages and disadvantages relative to each other. Nonintrusive traffic-monitoring technologies based on ultrasound, radar (Radio, Laser, and Photo), video and audio signals. All above present different characteristics in terms of robustness to changes in environmental conditions; manufacture, installation, and repair costs; safety regulation compliance, and so forth (Valcarce et al., 2004).

Traffic surveillance systems based on video cameras cover a broad range of different tasks, such as vehicle count, lane occupancy, speed measurements and classification, but they also detect critical events as fire and smoke, traffic jams or lost cargo. The problem of vehicle counting and classification is most commonly solved by deploying inductive loops. These loops are very intrusive to the road pavement and, therefore cost associated with these is very high. Most video analytics systems on highways focus on counting and classification. Using general purpose surveillance cameras for traffic analysis is demanding job. The quality of surveillance data is generally poor, and the range of operational conditions (e.g., night time, inclement, and changeable weather) requires robust techniques. The use of road side acoustic signal seems to be good approach for traffic monitoring and parameter estimation purpose having very low installation, operation and maintenance cost; low-power requirement; operate in day and night condition.

This paper focuses on use of acoustic signal for familiarizing the reader with techniques that are more progressively used in ITSs. To make this paper manageable, we concentrate here on vehicular speed estimation, density estimation and classification of ground vehicles and thus, do not consider the acoustic signal for underwater vehicles.

The remainder of the paper is organized as follows. We will first consider the vehicular acoustic signal in Section 2 which describe outlook of various traffic noise signals. A review of acoustic signal based research for vehicular speed and density estimation is presented in Section 3 to analyze the underlying approaches. The state of the art for vehicular classification is presented in Section 4. In Section 5, detailed discussions and an outlook to future research are provided.

\section{Vehicular Acoustic Signal}

A vehicular acoustic signal is mixture of various noise signals such as tyre noise, engine idling noise, noise due to exhaust, engine block noise, noise due to aerodynamic effects, noise due to mechanical effects (e.g., axle rotation, brake, and suspension), air-turbulence noise and the honks. The spectrum analysis of these vehicular acoustic signals composed of wideband and harmonics components. The mixture weighting of spectral components at any location is depends upon the traffic density condition and vehicle speed. In former case if we consider traffic density as freely flowing then acoustic signal is mainly due to tyre noise and air turbulence noise. For medium flow traffic acoustic signal is mainly due to wide band drive by noise, some honks. For heavy 
traffic condition the acoustic signal is mainly due to engine idling noise and several honks. In later case it is decided on the basis of whether accelerating, turning, decelerating, whether vehicle is in good mechanical condition or not. A typical vehicle produces various noise depends on its velocity, load and mechanical condition. In general, approximation can be done as vehicular acoustic signal is categorized as follows.

\subsection{Tyre Noise}

Tyre noise refers to noise produced by rolling tyre as an interaction of rolling tyre with road surface. The tyre noise is also considered as main source of vehicle's total noise at a speed higher than $50 \mathrm{kph}$ (Sandberg, 2001; Road Directorate, 2005). Tyre noise has two components: air noise and vibrational noise (Road Directorate, 2005; Sandberg and Ejsmont, 2002). The air noise is produced due to air being sucked in the rubber blocks of the tyre and due to sucked out of it. Air noise dominant in the frequency ranges between $1 \mathrm{KHz}$ to $3 \mathrm{KHz}$. On the other hand vibrational noise is produced due to contact between tyre threads and road surface texture or the pavement texture. It is dominant in the frequency range $100 \mathrm{~Hz}$ to $1000 \mathrm{~Hz}$. Considering the direction of vehicle movement the horn effect is observed which has directional pattern (Graf et al., 2002). This effect is generated by road and tyre, which forms a geometrical structure that amplifies the noise (amplification results in tyre noise component in the frequency range $600 \mathrm{~Hz}$ to $2000 \mathrm{~Hz}$ ), produced due to tyre-road interaction (Sandberg and Ejsmont, 2002; Graf et al., 2002; Kuo et al., 2002). The directivity of horn depends upon tyre geometry, tyre thread geometry, weight and torque of tyre. The total tyre noise power along with horn effect lies in the frequency range $700-1300 \mathrm{~Hz}$. Therefore, tyre noise play vital role in acoustic signal for speed around and above $50 \mathrm{kph}$ and in traffic density state estimation. Fig. 1 shows the graph of noise of tyre versus noise of vehicles, as for the vehicles moving with speed less than $50 \mathrm{kph}$ the noise of tyre is also less and as speed increases noise of tyre also increases.

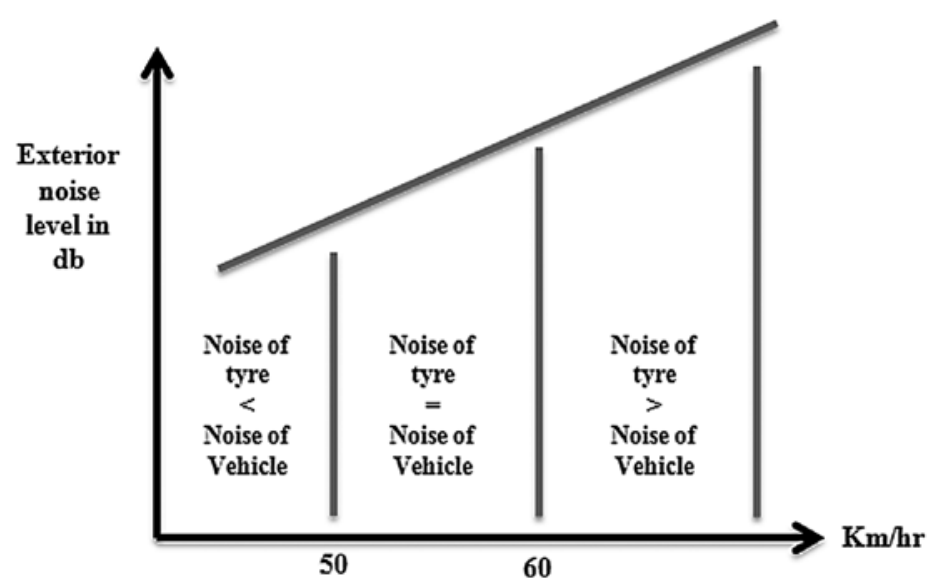

Fig. 1.

Noise of the Tyre vs Noise of the Vehicle 
The tyre noise is caused by three different factors:

- $\quad$ Tyre hitting ground (Fig. 2a);

- Vibration of air through tread pattern (Fig. 2b);

- Vibrations passing through tyre (Fig. 2c).

\subsection{Engine Noise}

Engine noise is produced due to internal combustion of engine. Engine noise contains a deterministic harmonic train and stochastic component due to air intake (Amman and Das, 2001). The fuel combustion in engine cylinder leads to deterministic harmonic train where lowest harmonic tone refers to cylinder fire rate (function of the engine's rounds per minute). On the other hand stochastic component is largely due to the turbulent air flow in the air intake, the engine cooling systems, and the alternator fans. This stochastic component is wideband in nature. The deterministic component has more power than stochastic component. The engine noise varies with speed and the acceleration of vehicle (Amman and Das, 2001; Cevher et al., 2009). A stationary vehicle produces distinct engine idling noise whereas moving vehicle produces different engine noise in correspondence with cylinder fire rate. In the recent years, manufacturers designs quieter engine to suppress the noise level. So engine noise might be strong on front side of car compared to other directions.

\subsection{Exhaust Noise}

The exhaust noise is produced due to entire exhaust system. The system goes from the engine combustion compartment through exhaust tubes to the exhaust muffler present at the back of the vehicle generating exhaust noise. The exhaust noise is directly proportional to load of the vehicle (Lilly, 2005). The exhaust noise is characterised by having power spectrum around lower frequencies. Exhaust noise is affected by turbo chargers and after cooler (Lilly, 2005; Kuo and Morgan, 1999).

\subsection{Air Turbulence Noise}

Air turbulence noise is produced due to the air flow generated by the boundary layer of the vehicle. It is prominent immediately after the vehicle passes by the sensor (e.g. microphone). It produces distinctive driveby-noise or whoosh sound. The air turbulence noise depends on the aerodynamics of the
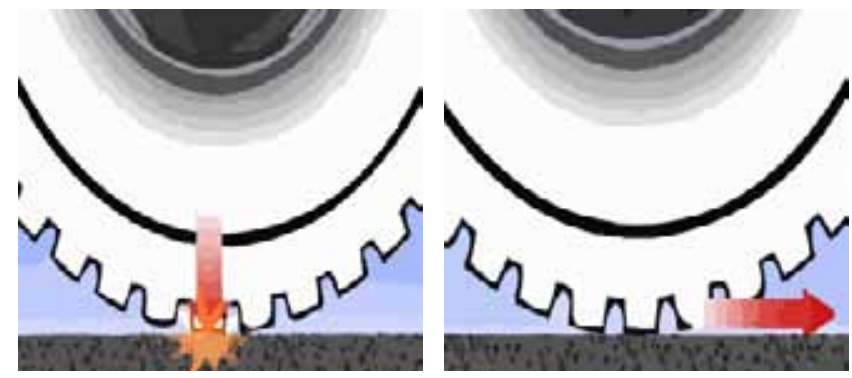

Fig. 2.

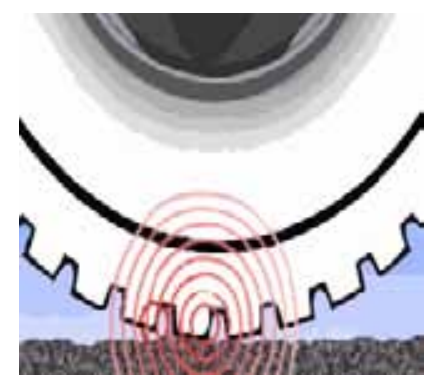

c) Vibrations Passing through the Tyre

Source: www.michelintransport.com

b) Vibration of the Air through the Tread Pattern, 
vehicle, wind speed and its orientation (Eskridge and Hunt, 1979; Sarigul-Klijn et al., 2001). For vehicular speed estimation case is considered when wind noise is much less than vehicle speed. For this perturbation are applied for the mean and the variance of the turbulent velocity components (KastnerKlein et al., 1998).

\section{Acoustic Signal based Approaches: Vehicular Speed and Density Estimation}

Today's urban environment is supported by applications of computer vision techniques and pattern recognition techniques including detection of traffic violation, vehicular density estimation, vehicular speed approximation, and the identification of road users. These techniques require data to be collected by various sensors such as magnetic loop sensors, speed gun, video cameras, etc. Currently magnetic loop detector is most widely used sensor for traffic monitoring in developing countries (Robertson and Bretherton, 1991). However, traffic monitoring by using these sensors still have very high installation and maintenance cost. Also these techniques require traffic to be orderly flow, traffic to be lane driven and in most cases it should be homogeneous.

Referring to the developing regions such India and Asia the traffic is non lane driven and highly chaotic. Highly heterogeneous traffic is present due to many two wheelers, three wheelers, four wheelers, auto-rickshaws, multi-wheeled buses and trucks, which does not follow lane. So it is the major concern of city authority to monitor such chaotic traffic. In such environment the loop detectors and computer-vision-based tracking techniques are ineffective. The use of road side acoustic signal seems to be good alternative for traffic monitoring purpose having very low installation, operation and maintenance cost. Several researchers have developed single-vehicle profile extraction techniques based on acoustic signal.

\subsection{Vehicular Speed Estimation}

Quinn (1996) and Couvreur and Bresler (1997) provides a theoretical description of single vehicle speed estimation using Doppler frequency shift. Assumption made that distance to the closest point of approach is known the solution can accommodate any line of arrival of the vehicle with respect to the microphone. The solution for speed estimation of single vehicle is more applicable as compared to several vehicles. In presence of several vehicles the interference is combined with acoustic waveforms.

Chen et al. (1997) and Chen and Sun (1997) reported sensing technique based on passive sound detection. These techniques utilizes microphone array to detect the sound waves generated by road side vehicles and are capable of monitoring traffic conditions on lane-bylane and vehicle-by-vehicle basis in a multilane carriageway. Correlation based algorithm are used (Chen et al., 1997; Chen and Sun, 1997; Chen et al., 2001), which extracts key data reflecting the road traffic conditions, e.g. the speed and density of vehicles. Chen et al. (2001) develops multilane traffic sensing concept based passive sound which is digitized and processed by an on-site computer using a correlation based algorithm. The system having low cost (installation, maintenance and operational), safe passive detection, immunity to adverse weather conditions, and competitive manufacturing cost. The system performs well for free flow traffic however for congested traffic performance is difficult to achieve.

Valcarce et al. (2004) exploit the differential time delays to estimate the speed. Pair of 
omnidirectional microphones was used and technique is based on maximum likelihood principle. It directly estimates car speed without any assumptions on the acoustic signal emitted by the vehicle. Lo and Ferguson (2000) develop a nonlinear least squares method for vehicle speed estimation using multiple microphones. Quasi-Newton method for computational efficiency was used. The estimated speed is obtained using generalized cross correlation method based on time-delayof-arrival estimates.

Mohan et al. (2008) estimates vehicle's speed using combination of smartphone features and basic honk signals. Simple Doppler frequency shift computations are done to estimate speed. Sen et al. (2010) used Doppler frequency shift rule with assumption that vehicle is moving in same direction as the straight line connecting vehicle to the microphone. In presence of multiple honking vehicles it is not clear that how two microphone distinct honk emitted by same vehicle. The experimental setup covers at two roads, on single lane one way and three lane bidirectional.

Cevher et al. (2007) uses single acoustic sensor to estimate vehicle's speed, width and length by jointly estimating acoustic wave patterns. Wave patterns are approximated using three envelop shape components. Results obtained from experimental setup shows the vehicle speeds are estimated as $(18.68,4.14) \mathrm{m} / \mathrm{s}$ by the video camera and $(18.60,4.49) \mathrm{m} / \mathrm{s}$ by the acoustic method. Cevher et al. (2009) estimate a single vehicle's speed, engine's rounds per minute (RPM), the number of cylinders, and its length and width based on its acoustical wave patterns. Wave patterns are determined using the vehicle's speed, the Doppler shift factor, the sensor's distance to the vehicle's closest-point-of-approach, and three (ES) components. Vehicle profile vector is estimated which provides a fingerprint for vehicle identification and classification (Ford F150, Chevy Impala, Honda Accord, Nissan Maxima \& Frontier, Mercedes E, Volvo 850 SW, Isuzu Rodeo and VW Passat). This system is only applicable for single vehicle and its type has been recognized.

\subsection{Traffic Density Estimation}

Urban areas are concerned with effective traffic signal control and traffic management. Time estimation for reaching from source to destination using real time traffic density information is major concern of city authorities. Referring to the developing geographical areas like Asia, the traffic is characterised be non lane-driven. In such condition traffic density estimation using magnetic loop detectors, speed guns and video monitoring seems to be best, but the installation, maintenance and operation cost associated with these approaches are very high. Use of road side acoustic signal seems to be an alternative for traffic density estimation. Kato (2005) proposed method for traffic density estimation based on recognition of temporal variations that appear on the power signals in accordance with vehicle passes through reference point. HMM is used for observation of local temporal variations over small periods of time, extracted by wavelet transformation. Experimental results show good accuracy for detection of passage of vehicles. Tyagi et al. (2012) classify traffic density state as Free flowing, Medium flow and Jammed. They consider short term spectral envelops features of cumulative acoustic signal, and then class conditional probability distribution is modelled on one of the three broad traffic density state (mentioned above). Experimental setup uses omnidirectional microphone placed at about $1.5 \mathrm{~m}$ height and cumulative acoustic signal 
is recorded at $16000 \mathrm{~Hz}$ sampling frequency. Bayes classifier is applied to classify traffic density state which results in $\sim 95 \%$ of accuracy, which is then improved by using discriminative classifier such as RBF-SVM. Compare with the existing computer vision and traffic monitoring system in Coifman et al. (1998), Cucchiara et al. (2000) and Kamijo et al. (2000) this technique is independent of light condition and works well for developing regions. Techniques in Mohan et al. (2008) and Sen et al. (2010) require accurate detection of honk signal to arrive at average speed. However, Tyagi et al. (2012) can't provide very accurate speed estimation compared to Cevher et al. (2009) and Couvreur and Bresler (1997).

\section{Vehicular Classification}

Problem of vehicular classification is example of pattern recognition theory. Acoustic signals collected by acoustic sensors are used to identify the type of moving ground vehicles. Typical classification process consists of sensing, class definition, feature extraction, classifier application and system evaluation (Fig. 3). Based on collected acoustic data feature vectors are extracted.

Sensing unit collects raw data in order to provide sensor node the information about traffic condition. Segmentation refers to separation of single vehicle imposes major restriction on acoustic classification system because traffic recordings are consist of signals from multiple vehicles which are mutually overlap. Feature extraction refers to extracting representative set of features which are able to distinguish different classes of vehicle. Duda et al. (2001) writes in "Pattern Classification": "The conceptual boundary between feature extraction and classification proper is somewhat arbitrary: An ideal feature extractor would yield a representation that makes the job of the classifier trivial; conversely, an omnipotent classifier would not need the help of a sophisticated feature extractor. The distinction is forced upon us for practical rather than theoretical reasons." Classification decides which class or category a given feature vector belongs. Broad categories are statistical and structural classifier.

Extensive research work has been carried out in vehicle detection and classification system, especially on battlefield vehicles.

\subsection{Multi-Category Ground Vehicle Classification}

Acoustic signal from ground vehicles contains wealth of information which can be used for vehicle classification. Engine and propulsion mechanism are two main source of acoustic emission (Lake, 1999). Ground vehicles can be sensed using various sensors (e.g. seismic, acoustic, magnetic, optical etc.). Among these

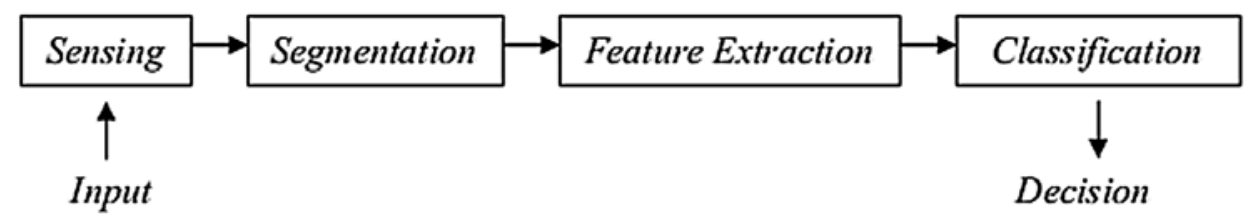

Fig. 3.

Typical Classification System

Source: Duda et al. (2001) 
sensors, seismic and acoustic sensors are characterized by their passive nature, non-line of sight sensing, long range, and low-power requirements and can be used either during day or at night (Damarla and Mirelli, 2006).

Choe et al. (1996) first compute the discrete wavelet transform of the acoustic signal in order to generate multiple resolution level spectrograms. The statistical parameters and the energy content of the wavelet coefficients from each spectrogram are considered to be features. Obtained features are compared with reference vehicle features to determine which class the input acoustic signal belongs to. MPP is used as classifier and accuracy obtained is $98.25 \%$. Continuous Wavelet Transform is used to determine unique signatures for different military vehicle types (Lopez et al., 1999). The feature vectors are constructed with 32 CWT filters. Very high classification results are achieved using multi layer neural network for 6 military classes. Wang and Qi (2002) classify 4 military vehicle classes using $\mathrm{k}$ - nearest neighbour and MPP. They not only use DWT coefficients, but also combine spectrograms and different moments to describe the average signal positions and spreads in time and frequency domain. Liu (1999) used the cochlear filter and cortical wavelet transform (CART) of the acoustic signal in order to obtain multiple resolution-level spectrograms in frequency domain. Vector quantization based clustering algorithms are used to classify vehicles. Accuracy obtained using this approach is $90 \%$.

Sampan (1997) extracts features considering energy envelop in time domain. Frequency bands from $2,7 \mathrm{kHz}$ to $5,4 \mathrm{kHz}$ and $4,5 \mathrm{kHz}$ to $5,4 \mathrm{kHz}$ are used in order to work in optimum frequency range. 30 features are derived heuristically and PCA is applied to achieve 24 features. A fuzzy logic system is used as classifier. Accuracy using fuzzy logic is $73-79 \%$ whereas for MLNN it is 95-97.5\%. Wellman et al. (1997) used three feature extraction methods: Power Spectrum Estimates (PSE), Harmonic Line Association (HLA), and Principal Component Analysis (PCA). An artificial neural network is used to classify vehicles. Accuracy obtained using HLA is $80-92 \%$ and for PSE: $10-94 \%$. Wu and Mendel (2007) demonstrate multicategory classification of battlefield ground vehicles using acoustic features. One fuzzy logic rule for each kind of vehicle was established. Three FLRBC architectures were proposed and evaluated against Bayesian classifier, which results in acceptable accuracy of $80 \%$. Damarla and Mirelli (2006) investigate tracking and classification of military and civilian vehicles using acoustic sensors. At each sensor node a distributed Bayesian classifier is used to determine the overall classification of each target. The distributed processing is robust to failures in sensor nodes unlike the centralized processing.

William and Hoffman (2011) classify military ground vehicles in two categories namely heavyweight and lightweight using multi-layer feedforward neural network. Implemented TDHA method enables sensor nodes to carry out the detection, extraction, and classification on-board. Classification rate reaches up to 89.67\%. Li et al. (2002) use Power Spectrum Estimation and Autoregressive modeling for feature extraction and classify two military classes using k-NNS, MLE, SVM. Simple cepstral coefficients without Mel-frequency warping are used which provide good class discrimination properties and may therefore also find application as feature vectors (Roberts et al., 2001). Classification was done using HMM and achieves accuracy of $96 \%$.

In developing nations such as India, traffic is mainly due to motorcycles. Anami et al. (2012) classify motorcycles into bikes and scooters 
based on the sounds produced by them. Where simple time domain and frequency domain features are used for classifier. Performance are analysed on ANN, KBC, DTW.

\subsection{Short Review of Domain Analysis}

Acoustic feature generation are mainly based on three domains: time, frequency, and both time-frequency domain.

\section{Discussion}

In urban environments, several monitoring objectives can be supported by the application of computer vision and pat-tern recognition techniques, including the detection of traffic violations, traffic density estimation, vehicle classification, vehicle count, vehicle speed estimation, etc.

Vehicular speed estimation begins with theoretical description of speed estimation using Doppler frequency shift (Quinn, 1996; Couvreur and Bresler, 1997). Followed by techniques based on passive sound detection and corresponding use of correlation algorithms to extract key data (Chen et al., 1997; Chen and Sun, 1997; Chen et al., 2001). Then techniques based on maximum likelihood principle (Valcarce et al., 2004), cross correlation method (Lo and Ferguson, 2000). Acoustic

\section{Table 1}

Vehicular Acoustic Feature Extractors and Classifiers

\begin{tabular}{|l|l|l|l|}
\hline Domain & Ref. & Feature Extractor & Classifier used \\
\hline \multirow{5}{*}{ Time } & (Sampan, 1997) & TE, PCA & Fuzzy Logic, MLNN \\
\cline { 2 - 4 } & (Chen et al., 2001) & Correlation based algorithm & \\
\cline { 2 - 4 } Frequency & (Succi et al., 1999) & HLA & NN \\
\cline { 2 - 4 } & (Wang and Qi, 2002) & HLA, DWT, STFT, PCA & k-NNS, MPP \\
\cline { 2 - 4 } & (Wellman et al., 1997) & HLA, PSE & NN \\
\cline { 2 - 4 } Time-Frequency & (Li et al., 2002) & PSE, AR Mod. & k-NNS, MLE, SVM \\
\cline { 2 - 4 } & (Looralahiyan and Kirby, 1998) & AR Mod. & MLNN \\
\cline { 2 - 4 } & (Choe et al., 1996) & DWT & MPP \\
\hline & (Wang and Qi, 2002) & CWT & MLNN \\
\hline
\end{tabular}

\section{Table 2}

Acronyms from Section 3 and 4

\begin{tabular}{|ll|ll|}
\hline TE & Time Energy Distribution & MLNN & Multi Layer Neural Network \\
\hline PCA & Principal Components Analysis & ANN & Artificial Neural Network \\
\hline HLA & Harmonic Line Association & k-NNS & k - Nearest Neighbor Search \\
\hline DWT & Discrete Wavelet Transform & MPP & Maximum Distance Approach \\
\hline STFT & Short Time Fourier Analysis & MLE & Maximum Likelihood Estimation \\
\hline PSE & Power Spectrum Estimation & SVM & Support Vector Machine \\
\hline AR Mod. & Autoregressive Modeling & RBF-SVM & Radial Basis Function- Support Vector Machine \\
\hline CWT & Continuous Wavelet Transform & & \\
\hline
\end{tabular}


wave patterns (Cevher et al., 2007; Cevher et al., 2009) proved to be excellent approach for vehicles speed, width and length estimation. Traffic is characterized by non-lane driven for developing regions, method in Kato (2005) based on recognition of temporal variations that appear on the power signals. Then use of short term spectral envelops features of cumulative acoustic signal (Tyagi et al., 2012). Extensive research work has been carried out for classification battlefield vehicles and few for urban. Where feature generation time domain (Sampan, 1997; Chen et al., 2001); frequency domain (Succi et al., 1999; Wang and Qi, 2002; Wellman et al., 1997; Li et al., 2002; Nooralahiyan and Kirby, 1998), timefrequency domain (Choe et al., 1996; Lopez et al., 1999; Wang and Qi, 2002) is carried out.

\subsection{Future Research}

In near future automated traffic monitoring play an essential role in improving road throughput and safety. At present scenario, most vision based monitoring techniques requires traffic data from large sensory network which is extremely expensive. Future traffic monitoring will analyze and assess traffic situations in real time under virtually all weather conditions. Robustness and adaptivity are key challenges which require numerous sensors to be installed at various locations to capture the traffic and estimate different traffic parameters. This diverse setting typically requires tedious sensor calibration and adapting the analysis algorithms to the observed scenes.

Another stream of research focuses on improving robustness by exploiting data from multiple sensors. Sensor fusion techniques can exploit the different characteristics of homogeneous or heterogeneous sensors. Clearer definitions of scenarios and applications are required to generate a more consistent body of work. New application areas are likely to emerge for traffic signal timings optimization using cumulative acoustic signals and also classification of motorcycles proved to be emerging area for research. Finally the classification systems can be extended in a way that extracted features are utilized as characteristic fingerprints, which affords tracking of vehicles over multiple sensor nodes.

\section{Conclusion}

We have presented a comprehensive review on acoustic signal based research in intelligent transportation systems, with a specific focus on urban environments and vehicle classification problems relates to battlefield environment. There is increasing scope in intelligent transportation system to adopt acoustic signal in traffic parameter measurement. The research on vehicular acoustic signal which is mixture of engine noise, tyre noise, noise due to mechanical effects etc., expands from vehicular speed estimation which is major concern of city authority in case of chaotic and non-lane driven environment, to density estimation. In later case estimation using magnetic loop detectors, speed guns and video monitoring seems to be best, but the installation, maintenance and operation cost associated with these approaches are very high. Hence use of road side acoustic signal seems to be an alternative, research shows acceptable accuracy for acoustic signal. Vehicular classification with acoustic signals proved to be excellent approach particularly for battlefield vehicles, and also for city vehicles.

Methods for vehicular speed estimation, density estimation, and classification have shown promising results, overcoming some of the issues of traditional methods, but are limited in different ways. Clearer definitions of scenarios and applications are required to generate a more 
consistent body of work. New application areas are likely to emerge for traffic signal timings optimization using cumulative acoustic signals and also classification of motorcycles proved to be emerging area for research. Finally, the presented classification systems can be extended in a way that extracted features are utilized as characteristic fingerprints, which affords tracking of vehicles over multiple sensor nodes.

\section{Acknowledgments}

Authors are thankful to the Director of GHRCE, for proving infrastructure to carry out research work and also to the anonymous reviewers for many helpful suggestions.

\section{References}

Amman, S.A.; Das, M. 2001. An Efficient Technique for Modeling and Synthesis of Automotive Engine Sounds, Industrial Electronics, IEEE Transactions. DOI: http://dx.doi.org/10.1109/41.904583, 48(1): 225-234.

Anami, B.S.; Pagi, V.B.; Magi, S.M. 2012. Comparative performance analysis of three classifiers for acoustic signal-based recognition of motorcycles using time- and frequency-domain features, Intelligent Transport Systems, IET. DOI: http://dx.doi.org/10.1049/iet-its.2011.0162, 6(3): 235-242.

Cevher, V.; Chellappa, R.; McCllelan, J.H. 2007. Joint Acoustic-Video Fingerprinting of Vehicles, PART I. In Proceedings of the IEEE International Conference on Acoustics, Speech and Signal Processing. DOI: http://dx.doi.org/10.1109/ICASSP.2007.366343, s: 745-748.

Cevher, V.; Chellappa, R.; McCllelan, J.H. 2009. Vehicle Speed Estimation Using Acoustic Wave Patterns, IEEE Transactions on Signal Processing. DOI: http://dx.doi.org/10.1109/TSP.2008.2005750, $57(1): 30-47$.
Chen, S.; Sun, Z.P. 1997. Traffic sensing by passive sound detection. In Proceedings of the VIII Conference on Sensors and their Applications, Glasgow, Scotland, U.K.

Chen, S.; Sun, Z.P.; Bridge, B. 1997. Automatic traffic monitoring by intelligent sound detection. In Proceedings of the IEEE Conference on Intelligent Transportation System. DOI: http://dx.doi.org/10.1109/ITSC.1997.660470, 171-176.

Chen, S.; Sun, Z.; Bridge, B. 2001. Traffic Monitoring Using Digital Sound Field Mapping, IEEE Transactions on Vehicular Technology. DOI: http://dx.doi. org/10.1109/25.966587, 50(6): 1582-1589.

Choe, H.; Karlsen, R.; Gerhart, G.; Meitzler, T. 1996. Wavelet based ground vehicle recognition using acoustic signals. In Proceedings of the SPIE, 2762: 434-445.

Coifman, B.; Beymer, D.; McLauchlan, P.; Malik, J. 1998. A real-time computer vision system for vehicle tracking and traffic surveillance, Transportation Research Part C: Emerging Technologies. DOI: http:// dx.doi.org/10.1016/S0968-090X(98)00019-9, 6(4): 271-288.

Couvreur, C.; Bresler, Y. 1997. Doppler-based motion estimation for wide-band sources from single passive sensor measurements. In Proceedings of the IEEE International Conference on Acoustics, Speech, and Signal Processing. DOI: http://dx.doi.org/10.1109/ICASSP.1997.604629, 5: 3537-3540.

Cucchiara, R.; Piccardi, M.; Mello, P. 2000. Image analysis and rule based reasoning for a traffic monitoring system, IEEE Transactions on Intelligent Transportation Systems. DOI: http://dx.doi.org/10.1109/6979.880969, 1(2): 119-130.

Damarla, T.R.; Mirelli, V. 2006. Distributed acoustic sensor data processing for target classification. In Proceedings of the SPIE-Unattended Ground, Sea and Air Sensor Technologies and Applications. DOI: http://dx.doi. org/10.1117/12.664585, 6231: 623105. 
Duda, R.O.; Hart, P.E.; Stork, D.G. 2001. Pattern Classification, John Wiley \& Sons Ltd.

Eskridge, R.E.; Hunt, J.C.R. 1979. Highway Modeling. Part I: Prediction of Velocity and Turbulence Fields in the Wake of Vehicles, American Meteorological Society. DOI: http:// dx.doi.org/10.1175/1520-0450(1979)018<0387:HMPI PO >2.0.CO;2, 18(4): 387-400.

Graf, R.A.G.; Kuo, C.Y.; Dowling, A.P.; Graham, W.R. 2002. On the horn effect of a tyre/road interface-Part I: Experiment and computation, Journal of Sound and Vibration. DOI: http://dx.doi.org/10.1006/ jsvi.2001.4238, 256(3): 417-431.

Kamijo, S.; Matsushita, Y.; Ikeuchi, K. 2000. Traffic monitoring and accident detection at intersections, IEEE Transaction on Intelligent Transportation Systems. DOI: http://dx.doi.org/10.1109/6979.880968, 1(2): 108-118.

Kastner-Klein, P.; Berkowicz, R.; Plate, E.J. 1998. Modelling of vehicle induced turbulence in air pollution studies for streets. In Proceedings of the $5^{\text {th }}$ Workshop on Harmonisation Within Atmospheric Dispersion Model-ling for Regulatory Purposes, Rhodes, Greece.

Kato, J. 2005. An Attempt to Acquire Traffic Density by Using Road Traffic Sound. In Proceedings of the 2005 International Conference on Active Media Technology. DOI: http://dx.doi.org/10.1109/AMT.2005.1505370, 353-358.

Kuo, C.Y.; Graf, R.A.G.; Dowling, A.P.; Graham, W.R. 2002. On the horn effect of a tyre/road interface-Part II: Asymptotic theories, Journal of Sound and Vibration. DOI: http://dx.doi.org/10.1006/jsvi.2001.4217, 256(3): 433-445.

Kuo, S.M.; Morgan, D.R. 1999. Active noise control: A tutorial review. In Proceedings of the IEEE. DOI: http:// dx.doi.org/10.1109/5.763310, 87(6): 943-973.

Lake, D. 1999. Efficient maximum likelihood estimation for multiple and coupled harmonics, Army Research Laboratory. 41 p.
Li, C.; Shimamoto, S. 2011. A Real Time Traffic Light Control Scheme for Reducing Vehicles $\mathrm{CO}_{2}$ Emissions. In Procedeeings of the 8th Annual IEEE Consumer Communications and Networking Conference - Emerging and Innovative Consumer Technologies and Applications. DOI: http://dx.doi.org/10.1109/CCNC.2011.5766627, 855-859.

Li, D.; Wong, D.K.; Sayeed, A.M. 2002. Detection, Classification and Tracking of Targets in Distributed Sensor Networks, IEEE Signal Processing Magazine. DOI: http:// dx.doi.org/10.1109/79.985674, 19(2): 17-29.

Lilly, J.G. 2005. Engine Exhaust Noise Control. Available from Internet: <http:www.ashraeregion7.org>.

Liu, L. 1999. Ground vehicle acoustic signal processing based on biological hearing models. M.Sc. thesis, Maryland University College Park Inst for Systems Research. 89 p.

Lo, K.W.; Ferguson, B.G. 2000. Broadband passive acoustic technique for target motion parameter estimation, IEEE Transactions on Aerospace and Electronic Systems. DOI: http://dx.doi.org/10.1109/7.826319, 36(1): 163-175.

Lopez, J.E.; Chen, H.H.; Saulnier, J. 1999. Target Identification Using Wavelet-based Feature Extraction and Neural Network Classifiers, Cytel Systems Inc., Hudson MA.

Mohan, P.; Padmanabhan, V.N.; Ramjee, R. 2008. Nericell: Rich monitoring of road and traffic conditions using mobile smartphone. In Proceedings of the 6th ACM conference on Embedded network sensor systems. DOI: http://dx.doi. org/10.1145/1460412.1460444, 323-336.

Nooralahiyan, A.Y.; Kirby, H.R. 1998. Vehicle Classification by Acoustic Signature, Mathematical and Computer Modelling. DOI: http://dx.doi.org/10.1016/ S0895-7177(98)00060-0, 27(9-11): 205-214.

Quinn, B.G. 1996. Doppler speed and range estimation using frequency and amplitude estimates, Acoustical Society of America. DOI: http://dx.doi.org/10.1121/1.413221, 98(5): 2560-2566. 
Road Directorate-Ministry of Transport. 2005. Noise Reducing Pavement, Road Directorate, Danish Road Institute Tech Report 141.

Roberts, W.J.J.; Sabrin, H.W.; Ephraim, Y. 2001. Ground Vehicle Classification using Hidden Markov Models, Atlantic coast technologies Inc., Silver Spring MD. 11 p.

Robertson, D.I.; Bretherton, R.D. 1991. Optimizing networks of traffic signals in real time-The SCOOT method, IEEE Transaction on Vehicular Technology. DOI: http://dx.doi.org/10.1109/25.69966, 40(1): 11-15.

Sampan, S. 1997. Neural fuzzy techniques in vehicle acoustic signal classification, Ph.D. dissertation, Department of Electrical Engineering, Virginia Polytechnic Institute and State University, Blacksburg, VA. 187 p.

Sandberg, U. 2001. Tyre/Road Noise-Myths and Realities. In Proceedings of the International Congress and Exhibition on Noise Control Engineering, Hague Netherlands. 22 p.

Sandberg, U.; Ejsmont, A.J. 2002. Tyre/Road Noise Reference Book. Kisa, Sweden: Infomex, SE-59040.

Sarigul-Klijn, N.; Dietz, D.; Karnopp, D.; Dummer, J. 2001. A computational Aeroacoustic Model for Near and Far Field Vehicle Noise predictions. The American Institute of Aeronautics and Astronautics Inc. $11 \mathrm{p}$.

Sen, R.; Raman, B.; Sharma, P. 2010. Horn-ok-please. In Proceedings of the ACM MobiSys, San Francisco, C. 137-150.

Succi, G.; Pedersen, T.K.; Gampert, R.; Prado, G. 1999. Acoustic Target Tracking and Target Identification - Recent Results. In Proceedings of the Conference on Unattended Ground Sensor Technologies and Applications. DOI: http:// dx.doi.org/10.1117/12.357130, 3713: 10-21.
Tyagi, V.; Kalyanaraman, S.; Krishnapuram, R. 2012. Vehicular Traffic Density State Estimation Based on Cumulative Road Acoustics, IEEE Transactions on Intelligent Transportation Systems. DOI: http://dx.doi. org/10.1109/TITS.2012.2190509, 13(3): 1156-1166.

Valcarce, R.; Mosquera, C.; Gonzalez, R. 2004. Estimation of road vehicle speed using two omnidirectional microphones: A maximum likelihood approach, EURASIP Journal on Advances in Signal Processing. DOI: http:// dx.doi.org/10.1155/S1110865704311133, 2004: 10591077.

Wang, X.; Qi, H. 2002. Acoustic target classification using distributed sensor arrays. In Proceedings of the 2002 IEEE International Conference on Acoustics, Speech, and Signal Processing. DOI: http://dx.doi.org/10.1109/ ICASSP.2002.5745661, 4: 4186-4189.

Wellman, M.C.; Srour, N.; Hills, D.B. 1997. Acoustic feature extraction for a neural network classifier Army Research Laboratory, Army Research Lab Adelphi Md. $25 \mathrm{p}$.

William, P.E.; Hoffman, M.W. 2011. Classification of Military Ground Vehicles Using Time Domain Harmonics' Amplitudes, IEEE transactions on instrumentation and measurement. DOI: http://dx.doi.org/10.1109/ TIM.2011.2135110, 60(11): 3720-3731.

Wu, H.; Mendel, J. 2007. Classification of Battlefield Ground Vehicles Using Acoustic Features and Fuzzy Logic Rule-Based Classifiers, IEEE Transaction on fuzzy systems. DOI: 10.1109/TFUZZ.2006.889760, 15(1): 56-72. 